https://helda.helsinki.fi

\title{
Analysing psychotherapy in practice
}

\section{Peräkylä, Anssi}

Cambridge University Press

2008

Peräkylä , A , Antaki , C , Vehviläinen , S \& Leudar , I 2008 , Analysing psychotherapy in practice . in E B A P E (ed.), Conversation analysis and psychotherapy . Cambridge University Press, Cambridge, pp. 5-25.

http://hdl.handle.net/10138/39209

publishedVersion

Downloaded from Helda, University of Helsinki institutional repository.

This is an electronic reprint of the original article.

This reprint may differ from the original in pagination and typographic detail.

Please cite the original version. 


\title{
$1 \quad$ Analysing psychotherapy in practice
}

\author{
Anssi Peräkylä, Charles Antaki, Sanna Vehviläinen, \\ and Ivan Leudar
}

\section{What are psychotherapies?}

The Oxford English Dictionary defines modern psychotherapy as "the treatment of disorders of the mind or personality by psychological or psychophysiological methods." Administering electroconvulsive shocks would, however, hardly count as psychotherapy; the common assumption is it that, in psychotherapies, the means of healing is talk. Not all talk is therapeutic, and the history of psychotherapy involves not just formulating new psychological theories but evolving new and distinct ways of talking with clients. This book is an effort to describe and to understand these distinct ways of talking.

Many psychoanalytic historiographies locate the invention of psychotherapy in Breuer's work with a patient they called Anna O. (described in Freud and Breuer's Studies on Hysteria, 1991/1895) at the end of nineteenth century. Anna $\mathrm{O}$. found that narrating her worries and fantasies helped to relieve her symptoms and she coined the phrase "the talking cure" to describe what she was doing. Freud used her case retrospectively to document the invention of psychoanalysis, which became the first form of psychotherapy. Rather soon, however, there emerged other ways of doing and thinking about "the talking cure," and at least since 1950s, the field of psychotherapy has been characterized by the multitude of (often rival) approaches. In psychotherapy with individual patients, client-centred psychotherapy gained influence in the 1950s (see e.g. Rogers, 1951), and cognitive-behavioural therapies have been increasingly popular since the 1970s (see e.g. Dryden, 2007). Alongside psychotherapies with individuals, group and family therapies based on psychoanalytic, system-theoretical, and later on social-constructionist ideas have been influential since the 1950s and 1960s.

Each school of individual, group, or family therapy is characterized by specific theoretical ideas about mind, behaviour, and social relations, and about the ways in which these may change. While, for example, psychoanalysis and psychodynamic therapies emphasize the importance of unconscious 
mental processes, cognitive therapies focus on adaptive and maladaptive interpretative schemes. In this book, we refer to such theoretical ideas, but they are not the main theme for us. What concerns us more is this: each different school of individual, group, or family therapy considers some interactional practices between therapists and patients to be the ones that promote change in the patient's mind, behaviour and social relations. Such practices may involve particular ways of asking questions, or of listening to and commenting upon the client's talk. One task of this book is to explicate, in greater detail than has been done before, some of these key interactional practices in specific forms of psychotherapy and group therapy.

There is, however, yet another major task for this collection. It is to point out and describe features of interaction that are part of psychotherapy but which the psychotherapeutic theories have not recognized or discussed. Psychotherapy is made possible by therapists and clients exerting their ordinary skills in social interaction as speakers, listeners, questioners, answerers, and so on. The contributions in this collection show some ways in which such ordinary interaction practices are made use of when conducting psychotherapy.

For all the contributors to this collection, conversation analysis (CA) provides (in varying degree) the research method and the central theoretical principles. (The details of CA will be described later in this chapter). In the CA perspective, the features of interaction that are specific to psychotherapies are firmly anchored in more generic features of social interaction which can be found in any human social conduct.

The conviction that psychotherapies are grounded in and related to broader everyday forms of life is not unique to CA. A similar kind of idea, in a rather different context, has been developed by scholars who draw upon the work of Michel Foucault (Foucault, 1967; 1977; Rose, 1996). They point out that psychotherapy depends on certain prior social practices. Relevant practices are those in which the modern reflective self and subjectivity originate. According to Foucault, self-monitoring and introspection are historically contingent, and originate in relatively recent practices of social surveillance. Psychotherapy presupposes these qualities in clients - that the person is divided into two related aspects: one that is public and visible and another that is private. If one is to participate in psychotherapy, one has to be able to inspect the innermost aspects of oneself and to do this just in the way pertinent to psychotherapy. There are concrete historical antecedents of psychotherapy in discursive practices such as confession.

As Foucauldian scholars have sought to show the embeddedness of psychotherapy in other historically contingent practices (such as confession) and competencies (such as self-monitoring), the contributions of this book 
will show the embeddedness of psychotherapy in generic interactional practices having to do with questions, answers, comments, and the like, and the related interactional competences that therapists and their clients employ.

\section{Studying psychotherapy: From case reports to the analysis of recorded interactions}

Since the formation of the first modern psychotherapeutic techniques at the turn of the twentieth century, the case report has been the default way of presenting and discussing data from psychotherapeutic encounters. Freud's case reports, such as "Dora" (Freud, 1905) or the "Rat Man" (Freud 1909) constitute classic examples. In case reports, all references to interactions between therapist and patient are based on the memory of the therapist. The case report often covers the whole treatment of the patient - i.e., it seeks to encapsulate what happened in possibly hundreds of sessions (plus, in most cases, the key events in the patient's biography).

Conversation analytic studies of psychotherapeutic interaction do not seek to compete with case reports. Rather, they seek to open up another kind of window for observing and understanding psychotherapeutic interaction. Rather than focusing on whole treatments and the ways in which these treatments have sought to redirect the biographical path of the patient, conversation analytic studies elucidate the second-by-second, or utterance-by-utterance, unfolding of psychotherapeutic sessions, with the aim of explicating the actual interactional patterns and practices through which psychotherapy gets done. Rather than relying on the memory of the participants, conversation analysts use audio and video recordings of actual psychotherapy sessions as their data.

However, conversation analysts are not the first researchers to use audio or video recorded data in the study of psychotherapeutic interaction. In the next section, a brief overview of earlier and parallel research will be given.

\section{Linguistic and social scientific studies of psychotherapy interaction}

Social scientific and linguistic analysis turned to psychotherapy as early as the 1950s. In fact, psychotherapy and psychiatric interviews provided the very first materials of the study of naturally occurring tape recorded or filmed interaction. The pioneering projects involved some of the leading anthropologists, linguists, and psychiatrists of the time, such as Gregory Bateson and Frieda Fromm-Reichman (see Kendon 1990, pp. 15-21).

The first major milestone in this line of research was the collaboration between an anthropological linguist (Charles Hockett) and two psychiatrists 
(Robert Pittenger and John Daheny). In a book called The first five minutes (Pittenger et al., 1961) they analysed in great detail the audio recording of the beginning of an initial interview with a patient of a psychotherapeutically oriented psychiatrist. Pittenger et al. describe the aim of their study in a way which is very close to the aims of the present collection. They wanted to "understand and describe what transpires in psychiatric interviews" (Pittenger, Hockett \& Danehy, 1961, p. 4). For them, asking the participants to describe the interview after it had been conducted was an unsatisfactory method of observation, because the participants' accounts would be selective, and offer inferences about the actual interview rather than a direct description of it. Instead, Pittenger et al. used tape recording, because

What concerns us ... is precisely the nature of the behaviour on which the inferences are based. . . We want to know about these things partly as a matter of basic scientific interest, and partly because such knowledge is obviously crucial in training new therapists. (Pittenger et al., 1961, p. 5).

Pittenger et al. came up with a detailed description, proceeding utterance by utterance, of the first five minutes of the interview. In searching for the implicit meanings of these utterances, the authors focused in particular on lexical choice (choice of words) and prosody (ways in which the utterances are delivered in terms of tone, volume, and speed). For example, in an exchange where the patient asks "may I smoke?" and the therapist responds "sure," the authors see two exchanges. Alongside the factual question, the patient is asking what kind of a situation they are in and what their relative status is. This other question is carried by the prosodic details of the patient's talk: breathiness, soft voice, high tone, and the specific way in which the word smoke is released (p. 40). Likewise, the choice of "sure" rather than "yes" in the therapist's response, along with its specific intonation contour, carry his response to this second question: he is conveying controlled surprise and implying that "the answer to your second question is that you don't have to ask permission here, and I'm surprised . . . that you should feel ... that you do" (p. 42).

What Pittenger et al. (1961) suggest about implicit meanings is intuitively most appealing. Their study is a genuinely explorative one: it offers insightful, if unsystematic, observations about a short segment of therapeutic interaction, without trying to make any generalizations about recurrent structures or practices in this interview, let alone in therapeutic interaction in general. The conclusions that the authors offer (pp. 228-250) have to do with the (then emergent) general theory and method of research on spoken interaction, rather than psychotherapy. The more systematic unravelling of the practices of psychotherapeutic interaction had to wait for subsequent studies. 
Another early research project was started by an anthropologist (Ray Birdwhistell) and a psychiatrist (Albert Scheflen) at the end of 1950s, but the publication of its main results was delayed until the early seventies (Scheflen, 1973). This research focuses on the filmed initial session of an experimental psychotherapy between a schizophrenic patient, her mother, and two experienced psychotherapists. Scheflen was particularly concerned about the uses of body posture and body movement during the psychotherapy session. He shows how the talk of the participants is coordinated with their body posture, producing nine basic positions such as "explaining," "passive protesting," "contending," and "defending" (see esp. p. 33). Likewise, Scheflen showed how the postures and postural changes of each participant are related to those of other participants. Through this work, Scheflen made a major contribution to the social scientific study of gesture. Moreover, he set his observations into the context of psychotherapeutic and family therapeutic theories, showing how the general theoretical principles of psychotherapy are realized through the therapist's uses of posture and body movement suggesting, for example, that rapport between patient and therapist is built largely through postural choices (pp. 237-264).

The next major milestone in the social scientific and linguistic analysis of psychotherapeutic interaction was the publication of William Labov and David Fanshel's Therapeutic discourse: Psychotherapy as conversation in 1977. This research was based on collaboration between a linguist (Labov) and a social work scholar (Fanshel). As in the studies described above, Labov and Fanshel examined a single segment of therapeutic interaction, in their case a 15 minute episode from the tape recording of an ongoing psychodynamic therapy with an anorexic patient.

Labov and Fanshel characterize their work as "comprehensive discourse analysis," and their analysis does indeed embrace various layers of the organization of verbal interaction, from phonological detail to overall "frames of discourse." At the core are what they call speech acts: the often implicit, multilayered actions that are performed through utterances. They single out four basic types of actions - metalinguistic action (initiating, continuing, or ending an action), representation, request, and challenge (Labov $\&$ Fanshel, 1977, pp. 60-65). Through the examination of the matrix of these actions, they address themes that are pertinent in the professional understanding of psychotherapy, such as emotion and repression.

Labov and Fanshel's study is rich in detail and insight, and the study is referred to time and again in interaction research. It draws a lively picture of the interaction between the patient and her therapist, as well as the patient's family interactions which are described in the patient's narratives. One important distinction Labov and Fanshel introduced was between 
descriptions of a state of affairs and the kind of knowledge participants are taken to have of it. Thus, an "A-event" is biographical or experiential information that the speaker has privileged access to, while others do not. A "Bevent" is a description of a matter in another speaker's experience, and the speaker thereby has limited access to it. Their system included other categories, but these two have turned out a crucial distinction even outside Labov and Fanshel's own approach. It has proved a central analytic dimension also for conversation analysts, under the title of "ownership of experience" (Peräkylä \& Silverman, 1991).

However, like The first five minutes, Labov and Fanshel's Psychotherapy as conversation does not seek to offer a systematic view of recurrent practices in psychotherapy. The typology of the four basic speech actions is very abstract and would apply to virtually any conversation. Like its predecessor, this study also offers as conclusions suggestions concerning the general theory and method of interaction analysis (Labov \& Fanshel, 1977, pp. 354-361).

The linguistic line of research on psychotherapy interaction was further continued and developed by Kathleen Warden Ferrara in her Therapeutic ways with words (1994). She points out (p. 4) the continuity between her study and that of Labov and Fanshel. However, unlike the studies mentioned above, she does not focus her study on a single segment of therapy talk, but uses a database of forty-eight hours of therapeutic interaction in the production of which six therapists and ten clients were involved. Ferrara's study explores the linguistic features of a number of recurrent "discourse strategies" in psychotherapy: personal experience narration, dream narration, repetition of the other's talk, construction of metaphors, and joint production of utterances. There is much in common between Ferrara's discourse analytic work and the conversation analytic studies presented in this collection. Ferrara, like most CA scholars, draws upon a large database and seeks to explicate recurrent practices of interaction through meticulous analysis of these data. The authors of this volume also explicate recurrent practices of psychotherapy; but to a greater extent than does Ferrara, they tie their observations to an understanding of the sequential structure of interaction and, hence, they seek to explicate the participants' orientations in producing the basic mechanisms of the psychotherapeutic interaction.

\section{Psychotherapy process research}

The studies described above drew the major part of their resources from social science and linguistics. They also contributed to these fields, by methodological and theoretical proposals concerning the study of spoken 
interaction. There is, however, also another current in the study of psychotherapy interaction. Rather than seeking to contribute to social science or linguistics, this stream of work seeks to address psychotherapists' concerns more directly. Basically, it seeks to find ways to describe how the change in the client takes place in and through the psychotherapeutic interaction. This line of investigation is often referred to as psychotherapy process research.

One traditional avenue to describe psychotherapy process involves coding and counting the participants' actions. Perhaps the most sophisticated undertaking in this direction is "verbal response mode" (VRM) analysis developed by Stiles (1992). The VRM coding scheme makes a distinction between eight types of utterance (such as "question," "reflection," or "interpretation") and yields global quantitative descriptions of psychotherapeutic sessions or segments of them. It has been used to document differences between psychotherapeutic approaches (such as "explorative" and "prescriptive") (Stiles, Shapiro \& Firth-Cozens, 1988), but researchers have failed to show correlates between the outcome (success) of the therapy and the therapist's verbal response modes (Stiles \& Shapiro, 1994; Stiles et al., 1988; ). Hence, the usefulness of research based on correlations of category frequencies with psychotherapy outcomes has been called into question by its main developer himself (Stiles, 1999). A good therapist is assumed to be responsive to the client's specific and momentary behaviours and this responsiveness cannot be measured by a fixed coding instrument.

The difficulties in attempting to describe therapeutic interactions in successful and less successful therapies have led psychotherapy researchers to seek other routes for understanding what happens in psychotherapy. These other routes seem to be more sensitive to the specific nature and tasks of psychotherapy, as well as to the specific characteristics of each patient. The assimilation model, developed by Stiles and his associates (Stiles, 2002; Stiles et al., 1990) seeks to understand psychotherapy as a process in which the client's relation to his or her particular problematic experience gradually changes. The problematic experience can involve, for example, painful memories, destructive relationships, or traumatic incidents. The model suggests that during the course of therapy, the clients "follow a regular developmental sequence of recognizing, reformulating, understanding and eventually resolving" such problematic experiences (Stiles 2002, p. 357). The assimilation model offers a way for categorizing segments of speech regarding the level of assimilation that they represent.

Many contemporary approaches in psychotherapy process research are similar to the assimilation model in terms of using recorded psychotherapy material as an index of the psychological change in the patient. Hence, for example, the "core conflictual relationship theme" method (CCRT) 
(Luborsky \& Crits-Christoph, 1997; Luborsky \& Luborsky, 1995) operates through coding the patient's accounts of his or her interactions with others. The coding is done by experienced clinical judges and aims at identifying patterns in how patients relate to others. The Referential Activity Scale (Bucci, 1995) seeks to describe the connections of the patient's emotional and other nonverbal experience with language, and operates through judges' ratings of properties (such as concreteness and imagery) of the patient's speech. Computerized analysis of speech (based on word counts) can also be used.

As a whole, psychotherapy process research involves an impressive effort to understand how psychotherapy works. Its practitioners have been both insightful and self-critical, and there is a constant search for more adequate ways for describing psychotherapy. Some studies have sought to give global characterizations of psychotherapeutic sessions, while others have focused on the ways in which the client's talk on specific topics indexes his or her inner state. Both approaches have successfully brought into light new aspects of the psychotherapeutic process. However, it seems that neither global characterizations (like VRM) nor methods which describe the change in the patient (like the assimilation model) are sensitive to the process through which the patient and the therapist together, and moment by moment create their psychotherapeutic sessions. This is where conversation analytic studies can make their contribution.

\section{Conversation analysis}

In this section we want to introduce the conversation analytic way of investigating social interaction - methodology through which we can develop new insight into the dynamics of psychotherapeutic practice. Conversation analysis (CA) was initially developed by Harvey Sacks (1992a; 1992b) and his colleagues (see e.g. Sacks, Schegloff \& Jefferson, 1974) at the University of California in the 1960s and early 1970s. It arose from, and is still closely connected to, the sociological tradition known as ethnomethodology (Garfinkel, 1967) which seeks to explicate processes of inference upon which the everyday social order is based. We will start by pointing out some issues that CA shares with at least some of the earlier approaches in psychotherapy research. Thereafter, we will outline the aspects of CA which make it a new and different approach in psychotherapy research.

The focus on action and a concern for detail are things that CA shares with earlier research on psychotherapy. For example, Labov and Fanshel (1977) studied "speech acts" such as representation, request, and challenge, and Stiles' (1992) "verbal response mode" analysis employs an actionoriented coding system that includes categories such as question, reflection, 
or interpretation. CA studies (in this collection and elsewhere) typically focus on specific actions, such as questions and answers (see chapter 5 by MacMartin), formulations (i.e. actions in which participants say in their own words what they understand that the others meant by their preceding utterances, see chapter 2 by Antaki), or therapists' interpretative statements and their patients' responses to them (see chapter 6 by Peräkylä and chapter 3 by Bercelli et al.). With the help of qualitative analysis of numerous instances of such actions, conversation analysts seek to explicate in detail how these actions are performed and responded to: what kind of words and syntactic structures are involved in them, what kind of presuppositions about the participants are created through them, and how the participants align or misalign while producing them.

Concern for detail is, as we say, an important feature that CA shares with many earlier studies on psychotherapeutic interaction. The analyses of Pittinger et al. (1961) and of Labov \& Fanshel (1977) were particularly rich in prosodic detail, and those of Scheflen (1973) in kinesic detail. Likewise, CA studies involve an effort to attend as much as possible to vocal and (when available in the data) visual aspects of the actions that are being studied. So, if you look through the pages of this book you will see that there is much concern for the fine detail of talk, ornamented with a filigree of notational symbols. The transcription symbols used in CA were initially developed by Gail Jefferson in the 1970s and involve notation for intonation, silence, sighs, hesitations, and the like. (The symbols are explained on page $\mathrm{X}$.) The actions people perform in conversation are complex, so it is no surprise to find that the details of the talk that produce those actions are complex to a corresponding degree.

Besides sharing some central concerns with earlier research on psychotherapeutic interaction, CA studies also attend to something that the earlier studies did not deal with systematically. An effort to understand the sequentiality of social action is the core of CA. CA studies attend to the ways in which single utterances are intrinsically related to the utterances that precede them and the utterances that come after them. Or, to put it more precisely, CA studies how interactants design their utterances in such a manner that makes these utterances intrinsically related to preceding and subsequent utterances. Utterances that arise from what happened just before and create conditions for what can happen next form social actions. "Questions" and "answers," "requests" and "responses," or "assessments" are examples of social actions in this CA perspective. CA studies on psychotherapy seek to understand how therapists and clients perform such sequentially organized social actions by designing their utterances in particular ways that establish particular relations between the co-interactants' utterances. 
CA has now built up - after forty years of effort since the pioneering work of Harvey Sacks - a collection of structures organizing the relations between actions which we can identify in almost any interaction. The most basic concept that CA uses to illuminate talk, is the "adjacency pair" (Schegloff \& Sacks, 1973). The idea is that by launching something that strongly projects a certain class of response (as, for instance, a question projects an answer and a request an acceptance) a speaker shows the next speaker what they are both doing at the moment, and will direct (or limit) what the next speaker can do next.

If the adjacency pair is the basic unit of talk, then how it is exploited is the first resource that we can study if we are interested in what people do (rather than simply in what words they speak). After the first part of an adjacency pair has been launched, the next speaker is at liberty to respond appropriately or not; but if they do not (and inspection finds that nonnormative responses will be marked by hesitation, a pause, and perhaps something like "well ...") then they will suffer - or exploit - the implications of so doing. Hence answering the question "Can you lend me that book?" with a brief pause and a "well . .." will mark the answer as not the expected one, and economically signal that the answer is "no." Empirical investigations demonstrate many such regularities in talk (across many languages) and indicate that, in Sacks' resonant phrase, there is "order at all points." Announcements get receipts, questions get answers, invitations get acceptances, and so on; the second utterance depends for its meaning on what has preceded it, and departures from these "expectables" are marked displaying orientation towards the normative order of conversation.

Many chapters of this book focus on actions which involve the structure of the adjacency pair. In a number of chapters (e.g. Chapter 3 by Bercelli et al., Chapter 5 by MacMartin, and Chapter 8 by Halonen) what are analysed are questions and answers. In some other chapters (e.g. Chapter 2 by Antaki, Chapter 6 by Peräkylä, Chapter 7 by Vehviläinen, and Chapter 9 by Leudar $e t$ al.) the analytic interest lies in the therapists' statement-formatted utterances that serve as "first-pair parts" and project an acceptance or rejection from the client. In all these chapters, any properties of the action of one participant (such as its word choice or the presuppositions about the participants that it carries) are examined in their relation to the actions of the other participants.

An adjacency pair involves a strict relation between two utterances. The sequential analysis of psychotherapy (or other type of interaction), however, encompasses relations that go beyond this basic structure. Any utterance in interaction - even when it is not produced as an element of an adjacency pair - "proposes a here-and-now definition of the situation to which subsequent talk will be oriented" (Heritage \& Atkinson, 1984, p. 5). Schegloff and Sacks (1973) named this generic property of utterances their 
"sequential implicativeness." Thus, for example, when a therapist is formulating (saying in his or her own words) what he or she understood that the patient was saying in his or her preceding utterance (see Chapters 2 by Antaki and 3 by Bercelli et al.), or when the therapist continues (extends) an utterance that the patient has started (see Chapters 6 by Peräkylä and 7 by Vehviläinen), the therapist's action is intrinsically tied and oriented to what the patient did in his or her utterance and also projects a delimited range of next utterance as the client's part. It is such retrospective and prospective relations between an action and its surrounding actions that the chapters of this book seek to explicate.

$\mathrm{CA}$ is, however, not only about relations between utterances that immediately follow one and precede another. The sequential implicativeness of individual utterances gives rise to, and serves as resource of, patterns that span over several turns (see Schegloff, 2007). Long and complex sequences can be built around a single adjacency pair through what Schegloff (2007) calls expansions. For example, in a pre-expansion, a particular adjacency pair serves as preparation for another adjacency pair to occur. A paradigmatic case involves a "pre-invitation": the question "are you free tonight" may serve as a preparation for an invitation to be issued (should the addressee be free). The delivery and reception of stories (Sacks, 1974) involves another example of a pattern that spans over several turns. Furthermore, in what Schegloff (pp. 195-216) calls sequences of sequences, successive sequences are linked - chaining of questions (Sacks, 1972, p. 343) where one party asks a series of questions of the other party is an example of this. In this book, patterns that extend over several turns are discussed, for example, in Chapter 3 by Bercelli et al. which explores the relations between therapists' questions, patients' answers, and the therapists' reinterpretative statements that sometimes follow the answers. In a similar vein, Chapters 6 by Peräkylä and 7 by Vehviläinen touch upon the ways in which therapists may prepare for their interpretative statements through a number of actions that precede them.

There is a yet larger scale of organization operative in interaction. Sequences that take place within a given encounter can be produced in such ways that connect them with past or present encounters (Button, 1991). For example, in openings and closings of conversations the participants show, in various ways, their orientation to what their relation has been and is expected to be. Moreover, a particular topic-e.g., a specific problem that is spoken about - can have a history over several encounters during which the participants' positions may change (Heritage \& Lindström, 1998). Such extended connectedness of encounters is of utmost importance in psychotherapy. In this collection, process of change over several sessions is addressed in Chapter 9 by Leudar et al. 
The analysis of sequentiality of action is thus the contribution that CA can make in psychotherapy research. But why should psychotherapists and psychotherapy researchers be interested in that contribution? It is the conviction of both the editors of this book and the authors of the chapters within it that sequential relations of actions are a major vehicle of the psychotherapeutic process. Any action of the therapist - be it a question, a statement, or something else - expresses an understanding of the patient's experience, and an understanding of how that experience can and possibly should be related to. (It does, of course, also propose an understanding of the therapist's own experience, which is of utmost importance for psychotherapy, but need not concern us at the moment.) These expressed understandings are achieved, mostly, through turn design - selection of words, descriptions, syntactical structures, as well as the perspectives and presuppositions that are built in the utterances. For example, Chapter 5 by MacMartin analyses therapists' questions in constructivist therapy, and shows how they are designed so as to convey an optimistic view about the patients' capacity to manage their lives. Likewise, the patients' actions - be they stories, answers, responses to interpretative statements, or the like - express comparable understandings of their own experiences. In MacMartin's Chapter 5, the patients' answers take stance to the questioners' optimistic presuppositions (and in her data, mostly misalign with them). Because the participants' actions are tied together by sequential implicativeness, the participants inevitably have to orient to and work with the understandings that they each bring about through their actions. The next action has to orient to the understandings that the first action brought forward. This work with understandings unfolds, literally, utterance by utterance. If things go well, this interplay of the participants' actions brings about a favourable change in the patients' ways of understanding and relating to their experiences. Sequential analysis is the microscope through which we will observe that process.

\section{Study of institutional interaction}

When Harvey Sacks and his colleagues started CA, they understood it as a programme for studying the properties and structures that underlie any social interaction. In the past twenty years or so, however, many conversation analysts have been involved in trying to understand specific kinds of interactions, ones in which the participants accomplish their specific, institutionally ascribed tasks. CA has been applied fruitfully to a variety of such institutional encounters: not only to the medical consultation, the news interview, and the classroom lesson, to list three of the more routine settings of everyday life; but also to such relatively unusual scenes such as the 
emergency call-centre, the police interrogation, and the psychic séance, among others (for overviews, see Arminen, 2005; Boden \& Zimmerman, 1991; Drew \& Heritage, 1992). Unlike everyday conversation among coequals, such "institutional" talk works to a more fixed order of talk in the design and distribution of turns (who gets to say what, when). What CA wants to do when analysing such interactions is to show how the people involved use these rules to transact their business, be it to pursue the answer to a question from an evasive politician (Clayman \& Heritage, 2002), to assess the caller's need for assistance in emergency calls (Zimmerman, 1992) or even to summon the presence of the dead from beyond the grave (Wooffitt, 1992).

Psychotherapy is one particular kind of institutional interaction. Before moving on to an account of earlier CA studies on psychotherapy, it will help if we examine in a bit more detail what CA studies have revealed about another specific type of institutional interaction. So, we will take the medical general practice consultation as an example for illustration. Like psychotherapy, general practice consultation involves interaction between a highly skilled specialist and a client who has come to seek help for personally meaningful problems that he or she cannot manage on her own. Unlike psychotherapy, medical consultation has been studied quite extensively by CA over the years. Heritage and Maynard (2006) provide us with an excellent review of the $\mathrm{CA}$ research on medical consultation, which allows us to see what kind of contribution CA might be able to give for understanding the encounter between a client and a professional.

The overall structural organization of the consultation is one of the key concerns in CA studies on medical consultation. Building upon the earlier work of Byrne and Long (1976), conversation analysts have shown how the clinician and the client orient themselves to an expectation that the acute consultation proceeds through a number of distinct phases: opening, presenting complaint, examination, diagnosis, treatment, and closing. This phase structure is not the analyst's stipulation of what ought to happen, but something that the participants themselves demonstrably orient to. For example, during the problem presentation, the patient may take actions that are associated with physical examination, diagnosis, or treatment (for example by asking about the possible cures), and thereby may indicate that the problem presentation is complete (Heritage \& Maynard 2006, p. 15).

Understanding the overall structural organization of the medical consultation has helped conversation analysts to set their questions and to contextualize the more detailed phenomena in consultation that they are studying. A large number of individual studies of general practice consultation have indeed explicated the ways in which the participants' conduct is organized in a particular phase, or at the juncture between particular phases. These 
studies focus on particular sequences through which different phases are accomplished. So, for example, Robinson (2006) describes doctors' uses of different question formats at the presenting complaint phase, Maynard and Frankel (2006) compare the ways in which good and bad news are delivered and responded to in diagnosis, Stivers (2005) describes the ways in which parents of patients who are children respond to the treatment recommendation, and West (2006) explicates some aspects of the collaborative closing of medical encounters. As a whole, in CA research on medical consultations, the studies on overall structural organization have provided, as it were, a large-scale map of the consultation, and many individual studies have provided smaller scale maps on key sites (the key sequences of each phase of the consultation) on that large-scale map. In result, these studies amount to a cumulative programme leading to an increasingly detailed and increasingly unified picture of the primary care consultation. Besides the social scientific interest, these studies also arise from practical concerns: their results have been useful in medical education as well as in interventions that seek to improve existing medical practice (Maynard \& Heritage, 2005).

\section{CA studies on psychotherapeutic interaction}

Harvey Sacks' life work formed the cornerstone for conversation analysis. He examined both so-called everyday conversations and institutional interactions: among them helpline calls received at a suicide prevention centre as well as an eleven-minute stretch of a group therapy session for four teenage boys (see Sacks 1992a, pp. 3-20 and pp. 268-280 respectively for his initial comments on these data). Sacks used everyday conversations and institutional interactions as data side by side, to examine the underlying structures and properties of all social interaction and social life therein. Sacks' lectures, using various data, show glimpses into corners of the social world that are more than relevant to psychotherapeutic settings. For instance, he explicated ways in which we organize our ways of referring to other persons in terms of their responsibilities to help us and others, means by which we maintain the fundamental presupposition of "ordinariness" of our experience, and how we deliver and respond to stories. However, Sacks' studies on these phenomena were not, at that time, presented under systematic rubrics such as "institutional talk" or "therapeutic interaction." His observations were not focused on the institutional character of the particular data he discussed. It was only many years after Sacks' untimely death that the conversation analytic research seeking to unravel specific psychotherapeutic practices began. In what follows, we will review some of the highlights of earlier CA studies on psychotherapy and related practices such as counselling and self-help groups. 
The first major study using CA to understand psychotherapy as a particular type of interaction was by Kathy Davis (1986) and took as its topic the therapist's ways of formulating clients' talk. Davis suggested that by such formulations, the therapist renders what was initially presented as a social or other "non-psychological" problem into a psychological one calling for psychotherapeutic intervention. Unlike most of the subsequent CA research on therapy, Davis then adopted a normative and critical stance towards the interactions that she studied. Therapists' formulations have remained in the CA researchers' agenda ever since; Chapter 2 by Antaki in this volume offers a summary.

The studies on formulations have tended to deal exclusively with one-toone psychotherapies. Group, family, and couple therapies have equally been of interest for conversation analysts. Also in this context, ways in which problems are described has been one of the key analytical issues. Thus, Edwards' (1995) analysis of talk in couples' counselling provides an angle on the way in which clients' talk is constructed for particular therapeutic purposes. He shows how clients present overtly disagreeing versions of their troubles to the counsellor, whose task it is to receive these versions impartially in order to find solutions to the problems. Edwards identifies a number of linguistic resources by which the speaker may describe another person's action in such a way as if it were following what he calls a "script," that is, as stemming from a culturally familiar routine with normative implications. He shows how couples design these script-like descriptions so that they carefully attend to their partner's counter-descriptions. Similarly, Edwards shows how speakers can choose among various categories of person in their narratives, so as to blame the other person, or head off blame for themselves. For example, a wife may shuttle between saying she was at a "girls' night out" (with its hint of fun and licentiousness) and describing the same event as "we sit around, one table full of married women" (1995, p. 30). Thus, as Edwards points out, speakers are very much aware of each other's countering versions and design their own versions so as to undermine them and defend their own version. Thus, there is a "rhetorical symmetry" between opposing versions of relationship troubles.

Systematic advances were also made, particularly in the 1990s, in the study of counselling interaction. An important set of counselling studies deals with HIV or AIDS counselling and guidance in Britain and the US (Kinnell \& Maynard, 1996; Peräkylä, 1995; Silverman, 1997). In HIV counselling, the main aim is to deliver information about HIV and AIDS, to advise people about safe sex and, thus, to prevent transmission of HIV. Another, more therapeutic, purpose of counselling is to help clients cope with related fears and problems. Due to this double function, the issues of advice-giving and delivery of expert views have a central role, but there is 
also a concern over how to create a space for the client's perspective and how to design the interaction so as best to serve the client in whatever situation she or he is in. One of the overarching themes in these studies is talk elicitation.

A core task for counsellors is to elicit personal narratives from their clients, often about delicate issues such as sexual behaviour or fears connected with HIV. Discreet exploration, studied initially by Bergmann (1992) in the context of psychiatric interviews, is prominent in many counselling settings and reported by several studies (Kinnell \& Maynard, 1996; Peräkylä \& Silverman, 1991). Devices of professional caution are typically used so as to downgrade the epistemological status of descriptions of issues that are "owned" by the recipient. This means that professionals speak cautiously and tentatively on matters that concern the client's experience, and imply in their talk that their knowledge is not authoritative. In counselling with children, talk elicitation poses a particular challenge. In a study focusing on counselling with children facing their parents' divorce, Hutchby (2002; cf. Potter 2006) showed a child used the claim "I don't know" strategically, "as a means for attempting to close down an undesired line of counsellor questioning" (p. 158). In Hutchby's data, the counsellor dealt with this resistance by modulating between playful and serious orientations to the repeated "I don't know" (see also Antaki, Chapter 2 this volume; Hutchby, 2007).

Peräkylä and Silverman (1991; Peräkylä, 1995) have examined talk elicitation in a setting in which counselling involves not only clients but also their partners or family members. These counsellors have adopted the Milan school family systems theory, which is a therapeutic theory about individuals and their problems as located in networks of relationships or "family systems." The counsellors use a particular interviewing technique called "circular questioning" to engage clients and their significant others in discussions about various issues. The authors show how all participants orient to the particular relationship that persons have to those experiences they “own" (Peräkylä \& Silverman, 1991), and how counsellors use particular questioning techniques to invoke ownership of experience and thus elicit client talk (Peräkylä, 1995).

Interaction in self-help groups such as Alcoholics Anonymous (AA) may share some key features with group and even individual psychotherapies. Clients' narratives are a central action in probably all therapies; particularly in AA. The sharing of monological stories and responding to others' stories through "second stories" (i.e. narratives that are presented as occasioned by prior stories and demonstrate similarity to them) are the interactional resource by which the recovery from addiction is accomplished (Arminen, 1998; 2004). Thereby, the sharing of personal stories constitutes a therapeutic 
practice. In the openings of the monological contributions in an AA meeting, there is a strong tendency to refer to other speakers' contributions, and thereby to show that one's topic is occasioned by a previous speaker's topic. Claiming similarity of experience, and demonstrating this similarity through providing an occasioned story are therapeutic practices by which AA members share their experiences, maintain sobriety, and construct their identities as recovering alcoholics (Arminen, 1998; 2004).

Arminen's work on the AA meeting also explicates the way in which the distinct, formal turn-taking order (1998, pp. 49-79) is oriented to and maintained by the participants, and how that, too, becomes the vehicle for therapeutic sharing. In the formal turn-taking order of AA, each speaker is entitled to one turn to talk about personal experience. Other speakers' turns are not countered or challenged. This format, with some variation, is known also in various group therapy settings where the participants orient to the expectation to share and identify with each other's experiences, although in settings where a professional is involved, he or she typically has different speaking rights than the clients and may perform various interventions so as to control the allocation of turn, to shape the narratives according to the therapeutic relevancies, or to confront the clients (Arminen \& Leppo, 2001; Jones \& Beach, 1995; Halonen, Chapter 8, this volume; Wootton, 1977).

Even this selective review of some key CA studies on psychotherapy and related practices probably indicates two things. One is the applicability of the CA method to research on psychotherapy. The researchers have been able to pin down and explicate some facets of interaction through which understandings of, and ways of relating to, experience are being worked with. Thus, the studies on formulations have demonstrated one particular practice for the therapist to subtly redirect the client's understanding of his or her problems; the studies on couple therapy have shown how the different versions of shared experiences are dealt with and made use of; the studies on HIV counselling have shown ways in which the clients are encouraged to verbalize their fears and worries; and studies on AA have shown how the similarity of the participants' experiences is interactionally demonstrated. The sequential analysis indeed seems to be capable of uncovering key aspects of the therapeutic process.

However, our limited review also shows that the progress of CA in the field of psychotherapy has, thus far, been somewhat unsystematic. No overall account of psychotherapy has been generated - that is to say, the CA research on psychotherapy has not produced anything that is comparable to the CA researchers' scheme concerning the overall structure of medical consultations. With regards to psychotherapy, CA researchers have investigated separate practices without gaining much understanding about the ways in which different practices are related to each other so as to 
produce a psychotherapeutic session as whole. There may be three reasons for this limitation of the research thus far. The most obvious one is that this line of research is its infancy - CA researchers have, for the most part, concentrated their efforts on other institutional settings. The second reason is the fact that psychotherapy is indeed a very varied practice. Unlike medical consultation which is rather uniform throughout the Western world, psychotherapy is characterized by multitude of (often competing) approaches. The ways in which psychotherapists of different schools engage the patients vary and, therefore, it is not likely that any single overall structure or other organizing principle of a psychotherapeutic session, applicable to all therapies, could be found. Finally, it is also possible that psychotherapy, or at least some psychotherapeutic approaches, is not organized in terms of distinct phases that would recur more or less similarly in each session. The key sequences - such as questions and answers, narrations and reception, formulation and response, and so on - may afford different ways of assembling them so as to form an entire session. But that is just one possibility. Basically, we do not know yet, and therefore new research, to be presented in this book and elsewhere, is needed.

This book will contribute to the enhancement of our understanding of psychotherapy by explicating a number of practices that are central for some central approaches of psychotherapy with individuals or groups. Some of these practices are ones that have been described in earlier research (for example, formulations analysed in Chapter 2 by Antaki) and the contribution of the book is to further and systematize the understanding. Others (such as the therapist's "noticings" about the patient's action analysed in Chapter 7 by Vehviläinen or lexical substitutions analysed in Chapter 4 by Rae) are such that have not been addressed in earlier CA studies. At the end of the book, in Chapter 11, the observations of the different practices provided by the individual chapters will be drawn together. The summary presented in that chapter takes us, we believe, as far as empirical research can at the moment go towards an overall picture of psychotherapeutic interaction.

\section{Conversation analysis addressing the concerns of psychotherapists}

In recent papers, Peräkylä and colleagues (Peräkylä \& Vehviläinen, 2003; Peräkylä, Ruusuvuori \& Vehviläinen, 2005) have suggested that for conversation analysis - as both a theory and an empirical investigation of interaction - it is relevant to recognize the existence of other theories of interaction and the way these theories may bear on the practices examined by CA. Professionals who meet clients - and treat, counsel, help, teach, or examine them - have theories, concepts and ideals that are related to their 
interactions with their clients. Practitioners understand their work (as well as related practices such as training or development) in terms of these theories, and much of the research around professional fields is conducted by reference to such theories. Therefore, it is essential that CA research has a grasp of these theories and ability to enter into a dialogue with them.

Peräkylä and Vehviläinen (2003) suggest that some of these theories can be conceptualized as "professional stocks of interactional knowledge" (SIKs). SIKs consist of organized and codified knowledge that concerns social interaction between professionals and clients. They can be found from professional texts, training materials, and codes of conduct. Peräkylä and Vehviläinen also propose that conversation analytic (CA) research can enter into a dialogue with the SIKs - extending, specifying or correcting the picture of interaction given by them.

In some contexts, a clear distinction can be made between theories or models that inform the interaction process ("interaction theories"), and other theories that seek to describe and assess other (non-interactional) aspects of the practice at hand, for instance, treatment theories within medical practices. In medicine, for instance, the interaction theories might involve propositions concerning ways of interviewing the patient or sharing information (e.g. "a patient-centred way of asking questions"). The treatment theory, on its part, involves propositions regarding the aetiology and cures of illnesses (Peräkylä et al., 2005; Peräkylä, Ruusuvuori \& Lindfors, 2007). In other types of settings, however, such a distinction is not as clear. For instance, in psychoanalysis, the propositions concerning the dynamics of psyche and the professional-patient interaction are intertwined and inseparable.

When CA results are put into dialogue with SIKs, in some cases the main contribution of CA turns out to be that of showing how a particular task or activity - recognized also by the SIK - is carried out in the turn-by-turn interaction and to explicate the interactional dynamics of the devices and structures used in that context. Chapters 6 by Peräkylä and 9 by Leudar et al. (regarding interpretations), 7 by Vehviläinen (regarding resistance) and 5 by MacMartin (regarding optimistic questions) represent such contributions. At times, the task of CA has been to explicate the workings of an action - or aspect of an interactional device - that the SIK had NOT recognized. In this book, such a contribution is made, for example, in Chapter 2 by Antaki (regarding formulations) and Chapter 4 by Rae (regarding therapists' lexical substitutions). And sometimes the findings of CA show that the actual practices and task of the institution in focus are quite different from the ones described by the SIK. CA can, thus, point at mismatches between the SIK and the interactional practices (Peräkylä \& Vehviläinen, 2003; Peräkylä et al., 2005). 


\section{Conversation analysis and questions about the outcome of the therapy}

Psychotherapists, their clients, and the funding agencies are inevitably concerned with effectiveness of psychotherapy. The methods of evidencebased medicine (EBM; see, e.g. Wessely, 2001) nowadays offer the standard way to address the questions concerning effectiveness. EBM typically involves operationalizing the desired outcome of therapy by means of independently defined variables and comparing experimentally how different interventions affect them. EBM has successfully renewed somatic medicine, but as applied to psychotherapy, it has its limitations. EBM is concerned about the (measurable) outcomes of specific therapeutic interventions, but not about what these interventions in themselves consist of. It treats psychotherapeutic interactions themselves as given, or as a black box, without trying to say what it is out of all that happens in these interactions that produces the change. Leudar et al. (2005) have argued that in imposing common operationalizations, EBM ignores unique aspect of different psychotherapies and in using statistics in comparing average outcomes in groups, the method ignores the central tenet of psychotherapy - that clients are individuals. Moreover, as Stiles (1999) points out, skilful therapists adjust their interventions to the momentary contingencies of the therapeutic process, and hence, the assumption about a standardized psychotherapeutic "input" is unjustified.

Perhaps partly for the aforementioned reasons, an index of the outcome of psychotherapy has not been centrally involved in the design of CA studies, in this collection or elsewhere. However, we can point out some ways in which CA research has been, or could be, relevant also in terms of outcome, and hence could also contribute to a more adequate approach of "evidence based psychotherapy." First, many CA studies are concerned what might be called the internal outcome of psychotherapeutic interventions. As they explicate recurrent sequences of actions in psychotherapy (such as questions and answers, formulations and responses, or interpretations and responses) they also elucidate the ways in which the therapists' actions in these particular contexts produce an effect in the patient. This does not lead to an overall picture concerning the success of the particular therapies or types of therapies, but it does involve a new insight into the specific mechanisms through which the change in the patient in these therapies takes place.

It could also be possible to use CA as a complementary method in evidence-based studies. The "black box" limitation of those studies could be remedied by using CA to describe objectively the interactions that took place in a trial, and then using this information to warrant the conclusion 
that the therapy in question was indeed the kind of specific therapy that it was supposed to be. A more radical alternative is to use CA to describe the change in the pattern of interaction that may take place as the therapy progresses. Here the ethnomethodologically informed CA that works with case studies is particularly useful. Chapter 10 by Streeck proposes this kind of approach, and Chapter 9 by Leudar et al. demonstrates some of its potential. Conversation analytic case studies focusing on entire therapeutic processes (dyads or groups) could show what, in terms of the patterns of interaction, changes in a successful therapy, thus complementing the more global, population-based picture sought after by evidence-based studies. Case studies of that kind, and possible collaboration with policymakers, are prospects for the future. At the moment, CA is establishing a distinct perspective on the fundamental interactional basis of psychotherapies, and beginning to show what can be learnt by its methods. 